\title{
Reduction of postoperative bleeding following operative treatment of proximal humerus fractures using a collagen sponge
}

\author{
Sylvia Doleschal ${ }^{1}$, Thomas Schmickal ${ }^{1}$, Alexander Schuh ${ }^{2}$ \\ ${ }^{1}$ Department of Trauma Surgery, Neumarkt Hospital, Neumarkt, Germany; \\ ${ }^{2}$ Research Unit Orthopedics and General Surgery, Neumarkt Hospital, Neumarkt, Germany; \\ *Corresponding Author: Alexander.Schuh@klinikum.neumarkt.de
}

Received 15 September 2010; revised 18 October 2010; accepted 20 October 2010

\section{ABSTRACT}

Aim of the present study is to investigate the efficacy and safety of $\mathrm{TachoSil}^{\circledR}$ to reduce afterbleeding and hematoma following operative treatment of proximal humerus fractures. In a prospective randomized study we included a consecutive series of $\mathbf{4 0}$ patients with a proximal humeral fracture in this study. All fractures were stabilized surgically with a fixed-angle "Philos plate" from May 2008 through May 2009. All patients were divided in two groups: Group I with plate osteosynthesis without TachoSil, Group II with plate osteosynthesis with TachoSil. For statistical analysis $\mathrm{Chi}^{2}$-Test and U-Test were used. There were 4 perioperative complications in group II and one complication in group I (Chi ${ }^{2}$-Test: $p=0.233$ ). In group II one hardware failure occurred due to osteoporosis requiring revision and reosteosynthesis. Another patient suffered from paralysis of the radial nerve which healed uneventfully. One superficial postoperative infection and one superficial hematoma required revision surgery, too. In this group no subfascial hematoma developed. One subfascial hematoma which required no revision occurred in group $\mathrm{I}$. Blood transfusion was required 2 times in group I and 3 times in group II (Chi ${ }^{2}$-Test: $p=0.549$ ). In sum in group II there was a significant lower blood loss for the subfascial drain, the region where TachoSil was applied. No adverse affects related to TachoSil could be detected. Tachosil was found to be safe and effective for reduction of postoperative bleeding following operative treatment of proximal humerus fractures. Further studies with larger sample size are required to confirm the efficacy of TachoSil $^{\circledR}$ in orthopedic surgery.
Keywords: Hematoma; TachoSil; Proximal Humerus Fracture; Plate Osteosynthesis; Reduction

\section{INTRODUCTION}

Proximal humerus fractures are increasingly common in the elderly [1]. The majority of proximal humerus fractures are minimally displaced and can be successfully treated non-operatively with early rehabilitation. Operative treatment methods range from head-preserving stabilization to total joint replacement depending on the extent of displacement and fragmentation. There are various surgical head-preserving methods with different kinds of plates, external fixation, intramedullary devices and $\mathrm{K}$-wire procedures, sometimes combined with the use of anchoring devices [1-6]. Several new locked plate devices have been developed because research suggests plates with attached (locked) screws may provide improved fracture stability and healing. Locking the screw to the plate mechanically recreates a point of cortical bone contact, which may be useful in the poor cancellous bone of the proximal humerus. Locking plates also have a preconfigured shape and screw direction, which may reduce hardware complications $[1,4]$. Postoperative complications include superficial postoperative infections (8$10.3 \%$ ) [4,7] and hematoma (4.6\%) requiring revision surgery [4]. TachoSil and its predecessor products, TachoComb $^{\circledR}$ and TachoComb H, have been used in a variety of surgical settings since being introduced in the early 1990s. TachoSil is indicated for supportive treatment in surgery for improvement of haemostasis, to promote tissue sealing, and for suture support in vascular surgery where standard techniques are insufficient. Clinical studies have shown that TachoSil is effective in achieving haemostasis after kidney or liver resection [8-13]. TachoSil ${ }^{\circledR}$ is a sterile, absorbable, haemostatic agent that consists of an equine collagen patch coated on one side 
with human fibrinogen and human thrombin. Unlike other fibrin sealants that require preparation before use, TachoSil is a ready-to-use fixed combination that is activated by moisture on application, providing adherence to the resection surface and haemostasis. The adhesive strength of TachoSil has been shown to be significantly higher than that of liquid fibrin glue and the effect of the fibrinogen and thrombin together with the mechanical support of the collagen patch provides a physiologically extensible and pliable liquid and air tight seal [9].

Aim of the present study is to investigate the efficacy and safety of TachoSil ${ }^{\circledR}$ to reduce afterbleeding and hematoma following operative treatment of proximal humerus fractures using an interlocking plate.

\section{MATERIAL AND METHODS}

In a prospective randomized study we included a consecutive series of 40 patients with a proximal humeral fracture. All fractures were stabilized surgically with a fixed-angle plate from May 2008 through May 2009. We included patients with displaced, unstable proximal fractures of the humerus, provided the humeral head was not stripped of soft-tissue attachments and was technically reconstructable. Patients were included independently of the fracture type if they had a mature skeleton and if there had been a delay between accident and surgery of not more than 10 days. Patients with open fractures or concomitant fractures of the ipsilateral distal humerus or elbow were not included. Also excluded were patients with allergic reactions against TachoSil ${ }^{\circledR}$ constituents, pregnant or lactating women, severe neurologic or psychiatric disorders, missing compliance and participation in other clinical trials.

8 men and 32 women with a median age of 74.15 (44.6-88.6) years were included.

Mean weight was $65 \mathrm{Kg}$ (53-98). Mean height was $166 \mathrm{~cm}$ (149-174). Mean body mass index was calculated 24.32 (19.49-33.13). All patientes were devided in two groups: Group I with plate osteosynthesis without TachoSil $^{\circledR}$, Group II with plate osteosynthesis with TachoSil $^{\circledR}$. Every second patient was included in group II.

Standard radiographs in two planes (AP and axial) were obtained for all patients and were used to plan the surgical procedure. CT-scan was reserved for special cases. Fractures were categorized with reference to the Neer classification [14]. In the Neer classification, there were 3 2-segment fractures, 15 3-segment fractures, and 22 4-segment fractures. The implanted devices were 40 fixed-angle implants "Philos plates" (Synthes $\mathrm{GmbH}$, Solothurn, Switzerland).

\subsection{Surgical Technique}

The patient was placed in beach-chair position on a radiolucent table with side placement of an image intensifier that would allow viewing of the humeral head in two planes. The approach was an anterior deltoid split. Fracture reduction was achieved through indirect manoeuvres and/or with the help of an elevatorium or K-wires used as joysticks for reduction of the shaft-head displacement, and with sharp bone hooks for reduction of the tuberosities. When anatomical reduction was obtained, insertion of the screws was performed. Additional tension band wiring was required in one case. After osteosynthesis one subfascial and one subcutaneous drain was applicated, in group II a TachoSil ${ }^{\circledR}$ sponge was applicated on the plate additionally. Postoperatively, the shoulder was immobilized in a sling for 7-10 days followed by active movement up to $90^{\circ}$ abduction and free flexion and retroversion for 4 weeks after surgery; then free, active mobilization was allowed. Duration of operation, perioperative and early postoperative complications such as hematoma and infection and blood loss collected in the drains were recorded as well. Blood loss collected in the drains was documented after 30 minutes (M 30), 60 minutes (M 60), 1 day (T01), 2 days (T02) and 3 days (T03) after the operation. The drains were removed on the third day.

For statistical analysis $\mathrm{Chi}^{2}$-Test and U-Test were used. The level of significance was set at $\mathrm{p} \leq 0.05$.

\section{RESULTS}

There were 4 peri-operative complications in group II and one complication in group I ( $\mathrm{Chi}^{2}$-Test: $\left.\mathrm{p}=0.233\right)$. In group II one hardware failure occurred due to osteoporosis requiring revision and reosteosynthesis. Another patient suffered from paralysis of the radial nerve on day 2 after the operation and recovered uneventfully. One superficial postoperative infection and one superficial hematoma required revision surgery, too.

In this group no subfascial hematoma developed. One subfascial hematoma which required no revision occurred in group I.

Blood transfusion was required 2 times in group I and 3 times in group II (Chi ${ }^{2}$-Test: $\left.\mathrm{p}=0.549\right)$.

Duration of the operation was $78.95 \pm 42.61$ minutes in group I and $72.63 \pm 20.93$ minutes in group II (U-Test: $\mathrm{p}=0.930$ ).

Table 1 shows the documented blood loss in the drains. In sum in group II there was a significant lower blood loss for the subfascial drain, the region where TachoSil $^{\circledR}$ was applied. (Figure 1) For the subcutaneous drains there was an obvious but not significant higher blood loss in group II. No adverse affects related to Ta$\mathrm{choSil}^{\circledR}$ could be detected. 
Table 1. Cummulated blood loss (*: statistical significant).

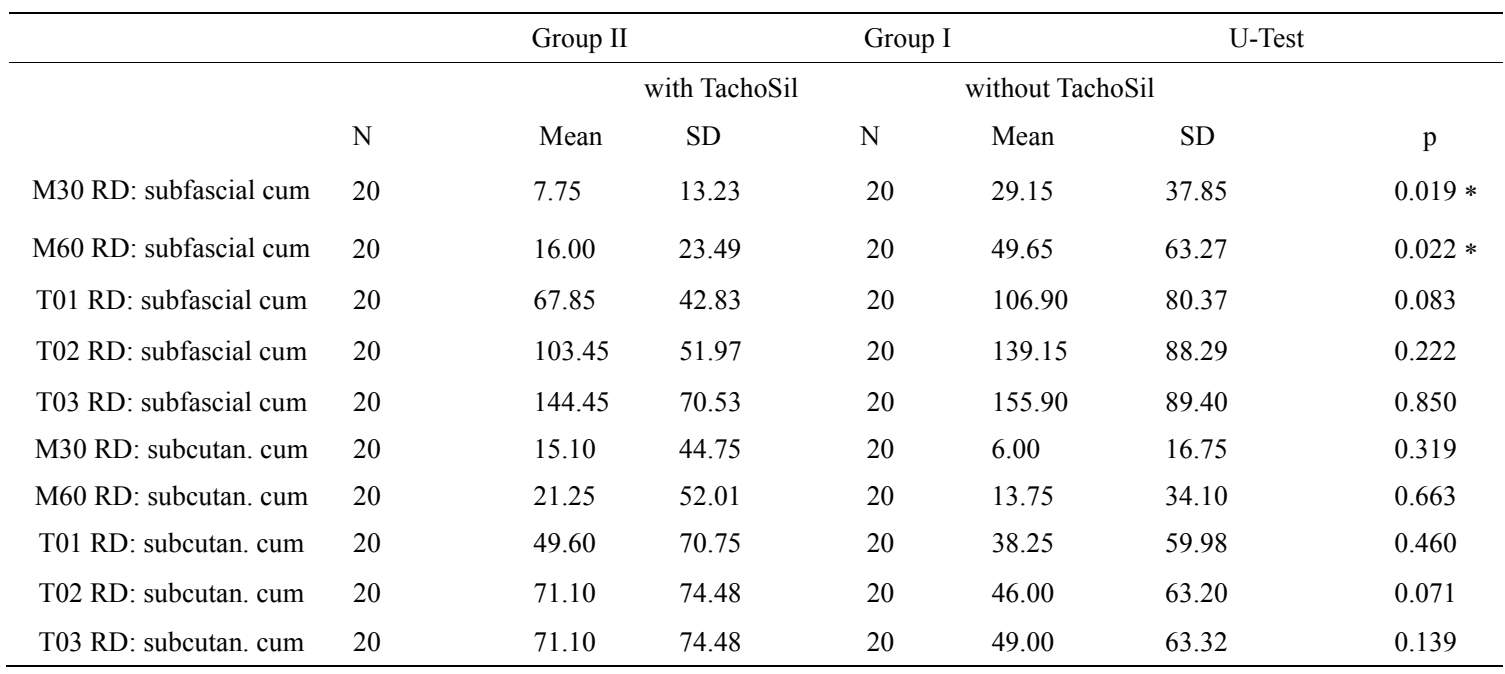

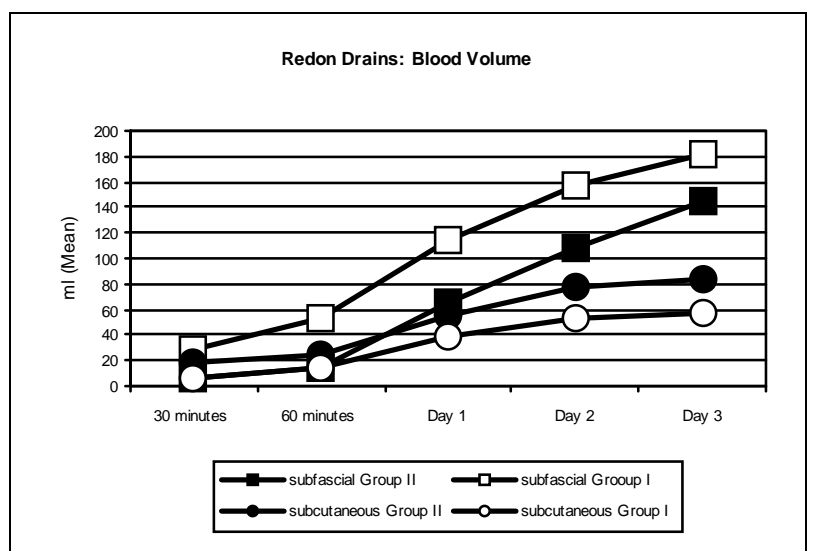

Figure 1. Cummulated blood loss collected in the drains.

\section{DISCUSSION}

The literature describes many options for treatment of displaced proximal humerus fractures. Treatment focuses on the displaced fracture fragments, since these may have limited vascularity and may benefit from reduction and fixation [4]. Locking plates with a preconfigured shape and screw direction, which may reduce hardware complications are well established [1-6]. Postoperative complications include pseudarthrosis, AVN, loss of correction, lesion of the radial nerve, superficial postoperative infections (8-10.3\%) [1,7] and hematoma formation $(4.6 \%)$ requiring revision surgery [1]. In our study TachoSil $^{\circledR}$ was used to provide fibrinogen and thrombin locally at the site of bleeding. Upon contact with fluid the clotting factors of TachoSil ${ }^{\circledR}$ dissolve and form a fibrin network, which glues the collagen sponge to the wound surface. Combining the clotting factors in a collagen patch provides a high concentration of clotting factors at the site where it is specifically needed [9]. In our study we could detect a significant lower blood loss of the subfascial drains 30 and 60 minutes after wound closure, which proves the effectiveness of $\mathrm{TachoSil}^{\circledR}$. There were no statistical significant differences of blood loss of the subfascial and subcutaneous drains in the further follow- up.

There were 4 peri-operative complications in group II and one complication in group I $\left(\mathrm{Chi}^{2}\right.$-Test: $\left.\mathrm{p}=0.233\right)$. In the TachoSil ${ }^{\circledR}$ group (group II) one hardware failure occurred due to osteoporosis requiring revision and re-osteosynthesis. Another patient suffered from paralysis of the radial nerve on day 2 after the operation and recovered uneventfully. One superficial postoperative infection and one superficial hematoma required revision surgery, too. The above mentioned complications are not related to TachoSil ${ }^{\circledR}$. In group I (without TachoSil ${ }^{\mathbb{B}}$ ) one subfascial hematoma occurred, which required no revision. Blood transfusion was required 2 times in group I and 3 times in group II (Chi ${ }^{2}$-Test: $\left.\mathrm{p}=0.549\right)$.

This study has one major limitation. In our prospective feasibility study only a small cohort of patients was included. In that way we can state that there is an obvious benefit in surgical treatment of proximal humerus fractures using TachoSil ${ }^{\circledR}$ in respect to blood loss in the region of the applied sponge (here: subfascial region). Therefore further studies with a larger sample size are required to confirm the efficacy of TachoSil ${ }^{\circledR}$. Only a larger study group may explain the obvious higher blood loss of the subcutaneous drains which could be shown in the current study.

\section{CONCLUSION}

TachoSil was found to be safe and effective for reduction of postoperative bleeding following operative treatment of proximal humerus fractures. Further studies with larger sample size are required to confirm the 
efficacy of TachoSil ${ }^{\circledR}$ in orthopedic surgery.

\section{REFERENCES}

[1] Helwig, P., Bahrs,C., Epple, B., Oehm, J., Eingartner, C. and Weise, K. (2009) Does fixed-angle plate osteosynthesis solve the problems of a fractured proximal humerus? A prospective series of 87 patients. Acta Orthopaedica, 80, 92-96.

[2] Erhardt, J.B., Roderer, G., Grob, K., Forster, T.N., Stoffel, K. and Kuster, M.S. (2009) Early results in the treatment of proximal humeral fractures with a polyaxial locking plate. Archives of Orthopaedic and Trauma Surgery, 129, 1367-1374.

[3] Foruria, A.M., Carrascal, M.T., Revilla, C., Munuera, L. and Sanchez-Sotelo, J. (2010) Proximal humerus fracture rotational stability after fixation using a locking plate or a fixed-angle locked nail: The role of implant stiffness. Clinical Biomechanics (Bristol, Avon), 25, 307-311.

[4] Friess, D.M. and Attia, A. (2008) Locking plate fixation for proximal humerus fractures: A omparison with other fixation techniques. Orthopedics, 31

http://orthosupersite.com/view.asp?rID $=34698$

[5] Röderer, G., AbouElsoud, M., Gebhard, F., Claes, L., Aschoff, A.J. and Kinzl, L. (2010) Biomechanical investigation of fixed-angle plate osteosynthesis of the proximal humerus. Unfallchirurg, 113, 133 -138.

[6] Südkamp, N., Bayer, J., Hepp, P., Voigt, C., Oestern, H., Kääb, M., Luo, C., Plecko, M., Wendt, K., Köstler, W. and Konrad, G. (2009) Open reduction and internal fixation of proximal humeral fractures with use of the locking proximal humerus plate. Results of a prospective, multicenter, observational study. Journal of Bone and Joint Surgery, 91, 1320-1328.

[7] Solberg, B.D., Moon, C.N., Franco, D.P. and Paiement, G.D. (2009) Surgical treatment of three and four-part proximal humeral fractures. Journal of Bone and Joint
Surgery, 91, 1689-1697.

[8] Anegg, U., Rychlik, R. and Smolle-Jüttner, F. (2008) Do the benefits of shorter hospital stay associated with the use of fleece-bound sealing outweigh the cost of the materials? Interactive CardioVascular and Thoracic Surgery, 2, 292-296.

[9] Bajardi, G., Pecoraro, F. and Mirabella, D. (2009) Efficacy of TachoSil patches in controlling Dacron suture-hole bleeding after abdominal aortic aneurysm open repair. Journal of Cardiothoracic Surgery, 4, 60.

[10] Frilling, A., Stavrou, G.A., Mischinger, H.J., de Hemptinne, B., Rokkjaer, M., Klempnauer, J., Thörne, A., Gloor, B., Beckebaum, S., Ghaffar, M.F. and Broelsch, C.E. (2005) Effectiveness of a new carrier-bound fibrin sealant versus argon beamer as haemostatic agent during liver resection: A randomised prospective trial. Langenbeck's archives of surgery, 390, 114-120.

[11] Haas, S. (2006) The use of a surgical patch coated with human coagulation factors in surgical routine: a multicenter postauthorization surveillance. Clinical and Applied Thrombosis/Hemostasis, 12, 445-450.

[12] Rickenbacher, A., Breitenstein, S., Lesurtel, M. and Frilling, A (2009) Efficacy of TachoSil a fibrin-based haemostat in different fields of surgery-A systematic review. Expert Opinion on Biological Therapy, 9, 897907.

[13] Siemer, S., Lahme, S., Altziebler, S., Machtens, S., Strohmaier, W., Wechsel, H.W., Goebell, P., Schmeller, N., Oberneder, R., Stolzenburg, J.U., Becker, H., Lüftenegger, W., Tetens, V. and Van Poppel, H. (2007) Efficacy and safety of TachoSil as haemostatic treatment versus standard suturing in kidney tumour resection: A randomised prospective study. European Urology, 52, 1156-1163.

[14] Neer, C.S. (1970) Displaced proximal humeral fractures. I. Classification and evaluation. Journal of Bone and Joint Surgery, 52, 1077-1089. 